\title{
Actividad antioxidante y marcha fitoquímica de los capítulos de Tagetes filifolia Lag. "pacha anís"
}

R. Sánchez-Humala ${ }^{1,3}$, A.M. Ruiz-Briceño ${ }^{1,3}$, C.G. Ruiz-Burneo ${ }^{1,3}$, G.M. Ruiz-Castro ${ }^{1,3}$, D.R. Sairitupac-Paredes ${ }^{1,3}$, L.G. Aguirre ${ }^{1,2,3}$, A. Salazar-Granara ${ }^{1,2,4}$, B. Loja-Herrera ${ }^{1,5}$

\section{RESUMEN}

Objetivo: Estudiar la actividad antioxidante y marcha fitoquímica de los capítulos de Tagetes filifolia Lag. "pacha anís“. Materiales y métodos: Estudio de tipo experimental en el cual se empleó $5 \mathrm{~kg}$ de los capítulos de la planta medicinal Tagetes filifolia lag., provenientes de Junín. Se usó el método de cribado fitoquímico de Olga Lock para la marcha fitoquímica y el método DPPH para la determinación de la actividad antioxidante. Se dividió la muestra en 3 grupos: etéreo, alcohol etílico y agua destilada a concentraciones de 100,50 y $5 \mu \mathrm{g} / \mathrm{ml}$.

Resultados: Se encontró fenoles en cantidades abundantes tanto en el extracto en agua destilada como en el extracto en alcohol etílico, además este último tuvo cantidades moderadas de quinonas. Por otro lado, el extracto en alcohol etílico fue el que presentó el mayor porcentaje de captación de radicales libres $(91.26 \%)$ a una concentración de $100 \mu \mathrm{g} / \mathrm{ml}$, similares resultados se encontró con el extracto etéreo (88.94\%) y el extracto en agua destilada (75.58\%).

Conclusiones: Los principales componentes químicos fueron fenoles y quinonas. El mayor efecto antioxidante se obtuvo del extracto etanólico de la planta Tagetes filifolia a una concentración de $100 \mu \mathrm{g} / \mathrm{ml}$.

Palabras clave: Composición química; antioxidantes; tagetes.

\section{Antioxidant activity and phytochemical screening of capitula of Tagetes filifolia Lag. or "pacha anís"}

\section{ABSTRACT}

Objective: To study the antioxidant activity and phytochemical screening of capitula of Tagetes filifolia Lag. "pacha anís".

Materials and methods: An experimental study which used $5 \mathrm{~kg}$ of capitula of a medicinal plant called Tagetes filifolia Lag. collected from the Department of Junín, Peru. The phytochemical screening method proposed by Olga Lock, M.D. was used for the phytochemical screening and the DPPH method for the determination of the antioxidant activity. The sample was divided into 3 groups: ether, ethyl alcohol and distilled water at concentrations of 100,50 and $5 \mu$ g/ml, respectively.

Results: Abundant amounts of phenols were found in both the distilled water extract and the ethyl alcohol extract, and the latter had moderate amounts of quinones. Besides, the ethyl alcohol extract showed the largest percentage of freeradical scavenging $(91.26 \%)$ at a concentration of $100 \mu \mathrm{g} / \mathrm{ml}$. Similar results were found in the ether extract (88.94\%) and the distilled water extract (75.58\%).

Conclusions: The main chemical components were phenols and quinones. The highest antioxidant effect was obtained from the ethanolic extract of Tagetes filifolia Lag. at a concentration of $100 \mu \mathrm{g} / \mathrm{ml}$.

Keywords: Chemistry; antioxidants; tagetes.

1. Unidad de Medicina Tradicional, Centro de Investigación de Medicina Tradicional y Farmacología ( CIMTFAR), Instituto de Investigación de la Facultad de Medicina Humana de la Universidad de San Martín de Porres (FMH-USMP). Lima, Perú.

2. Sociedad Científica de Estudiantes de Medicina Humana de la Universidad de San Martín de Porres. (SOCIEM-USMP)

3. Estudiante de Medicina Humana de la Universidad de San Martín de Porres, Investigador Junior.

4. Doctor en medicina, Médico-Cirujano, Profesor Investigador.

5. Doctor en Botánica, Bióloga, Profesora Investigadora. 


\section{INTRODUCCIÓN}

La Organización Mundial de la Salud (OMS), reconoce a la medicina tradicional (MT), como un sistema de salud, el cual está basado en diversas prácticas terapéuticas, entre ellas el uso de plantas medicinales ${ }^{(1)}$.

El Perú cuenta con un sistema de medicina tradicional, el cual incluye el uso de plantas medicinales nativas; asimismo, Perú es un país mega diverso al cual se le reconocen alrededor de 2000 especies de plantas con propiedades medicinales ${ }^{(2)}$.

La Tagetes filifolia (pacha anís) es una hierba aromática, nativa de América, y cuya extensión abarca países desde México hasta Argentina; en el Perú se encuentra en Amazonas, Ancash, Cajamarca, Cusco, Huánuco, La Libertad y Lambayeque ${ }^{(3-5)}$.

Estudios sobre el uso local de esta planta en poblaciones mexicanas indican que ésta tiene actividad analgésica y descongestionante ${ }^{(6)}$. El uso tradicional indica que la cocción de las partes aéreas ayuda en los cólicos digestivos y menstruales mientras que la infusión se usa para la diarrea (7); la cocción de las hojas y tallos, como antipsicótico y tusígeno; su aceite, como descongestionante de las vías aéreas ${ }^{(8,9)}$. En Perú, un estudio realizado en el callejón de Huaylas encontró 33 plantas medicinales de uso tradicional, dentro de ellas se reportó que Tagetes filifolia lag, fue usada por la población para contrarrestar dolor gastrointestinal ${ }^{(10)}$.

Esta planta se encuentra muy estudiada a nivel agro botánico, por su efecto pesticida sobre ciertos insectos, como la mosquita blanca de México, y su poco efecto toxico sobre el ser humano ${ }^{(11,12)}$. Su actividad insecticida también ha sido estudiada en el ámbito médico, por ejemplo, un estudio experimental utilizó los extractos de la raíz, las flores y las hojas de 3 especies de Tagetes sobre las larvas e insecto adultos de Aedes aegypti y Anopheles sthepensi, encontrando una alta toxicidad del extracto de T. minuta sobre estos, mientras que hubo mala respuesta con el uso de los aceites esenciales ${ }^{(13)}$. Por otro lado se ha investigado sobre el efecto tóxico de esta planta frente al piojo humano Pediculus humanus capitis ${ }^{(14)}$.

En cuanto a su efecto biológico, investigadores peruanos han estudiado el efecto de esta planta sobre la fertilidad, aplicando el sub extracto metanólico de hojas de anisillo en ratas Holtzmann. Llegaron a la conclusión que en ratas macho no afecta la producción de testosterona, mientras que en ratas hembras produce aumento de la gravidez ${ }^{(12,15)}$.

Por ende, los efectos de la planta podrían deberse a sus compuestos fitoquímicos. Se ha estudiado la marcha fitoquímica de su aceite esencial siendo cis-anethole, estragol, menthone e isomenthone, metilchavicol, B-cariofileno, linalol, piperitone, los más resaltados $(4,16,17)$. De estos compuestos, se ha encontrado que el estragol previene el edema y la inflamación por su actividad sobre los mediadores de fase tardía de la inflamación, inhibiendo directamente al Óxido Nítrico e, indirectamente, a la producción de prostanoides ${ }^{(18)}$. Además, este componente puede actuar localmente bloqueando los potenciales de acción en las neuronas de los ganglios de las raíces dorsales de ratas ${ }^{(19)}$.

La importancia de conocer la actividad antioxidante de esta planta recae en su posible potencial como recurso antioxidante, al reducir el estrés oxidativo. Por lo tanto, puede servir de gran apoyo en el tratamiento de enfermedades crónicas como diabetes, hipertensión arterial y el cáncer; cuya fisiopatología está asociada con la sobreproducción de especies reactivas de oxígeno ${ }^{(20)}$.

Hasta el momento la bibliografía se ha enfocado más en el estudio del aceite esencial de esta planta, (10) conociéndose poco sobre otros metabolitos secundarios como flavonoides, fenoles y alcaloides; y, también, sobre la actividad antioxidante de sus diferentes partes anatómicas. Nuestro estudio mejorará el conocimiento fitoquímico y la farmacopea peruana sobre el Tagetes filifolia.

\section{MATERIALES Y MÉTODOS}

\section{Lugar de ejecución}

El presente estudio fue ejecutado en el Laboratorio de Farmacología del Instituto de Investigación de la Facultad de Medicina Humana, Universidad de San Martín de Porres.

\section{Muestra vegetal}

La muestra de los capítulos fue recolectada en mayo del 2013 en el distrito de Matahuasi - Concepción (Junín) a 3443 m.s.n.m.

Para el estudio taxonómico, durante la colecta, se siguió el método de Cerrate E, $1969^{(21)}$; mientras que para el estudio químico, se siguió el método de Ramagosa, $2001^{\text {(22). }}$. Además, se usó el sistema de clasificación según Cronquist A, $1981^{(23)}$, para el ordenamiento taxonómico de la planta.

Clasificación según Cronquist, A. 1981

\begin{tabular}{|rc|}
\hline \multicolumn{1}{|c|}{ Reino } & Plantae \\
\hline División: & Magnoliophyta \\
\hline Clase: & Magnoliopsida \\
\hline Subclase: & Asteridae \\
\hline Orden: & Asterales \\
\hline Familia: & Asteraceae \\
\hline Género: & Tagetes L \\
\hline Especie: & Tagetes filifolia lag. \\
\hline
\end{tabular}


Actividad antioxidante y marcha fitoquímica de los capítulos de Tagetes filifolia Lag. "pacha anís"

Para la determinación de la planta "pacha anís" se emplearon claves y descripciones de la bibliografía nacional y extranjera, siendo de gran valor "La Flora de Perú" de Macbride, 1936-197 (24); Weberbauer, $1945^{(25)}$; Tovar, $1990^{(26)}$; entre otros.

La confirmación de nuestros ejemplares se realizó mediante comparación con otros del Herbario de la Universidad Nacional Mayor de San Marcos y de la Universidad Nacional Agraria La Molina; asimismo, se consultó con especialistas nacionales y extranjeros.

Para la selección de las plantas se usaron los siguientes criterios de inclusión: plantas medicinales pertenecientes a la especie Tagetes filifolia en etapa de floración para poder obtener los capítulos. Además, éstas debieron tener buena apariencia física y no estar contaminadas con mohos u otros agentes nocivos.

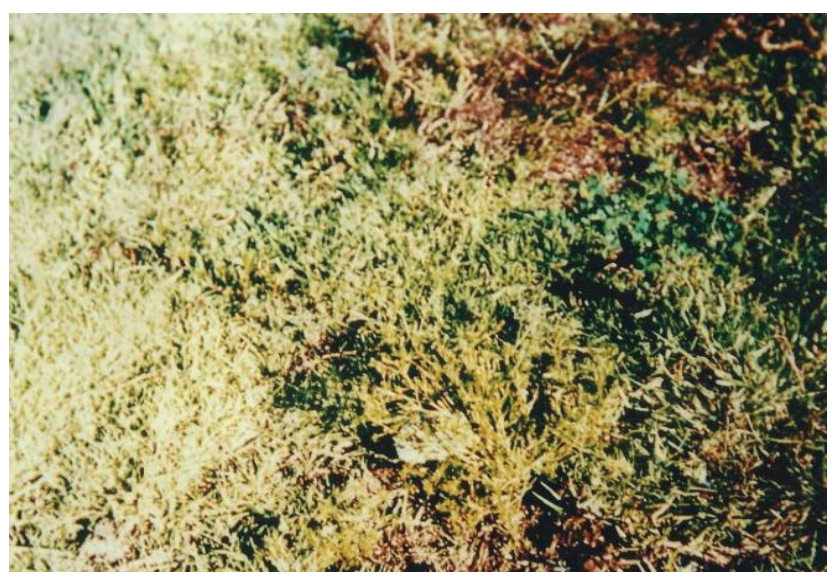

Figura 1. Tagetes filifolia Lag. (Tomado de Berta Loja Herrera)

\section{MUESTRA QUÍMICA:}

\section{Materiales químicos (reactivos)}

Se usó ácido ascórbico de Fisher (tipo Ácido ascórbico USP; procedencia México; marca JT Baker; fecha de expiración 2017), DPPH- 2,2-difenil-1-picrilhidrazilo (procedencia Estados Unidos; marca Sigma Aldrich; fecha de expiración 2018), metanol (tipo absoluto; procedencia México; fecha de expiración 2018), etanol (procedencia México; marca JT reactivo Baker; expira 2018) y agua destilada (agua para HPLC, procedencia México, JT Baker, fecha expiración 2017).

\section{Equipo}

Se usó un rotavapor modelo Laborota 4003 (Hieldolph); un espectrofotometro UV 2550 (Shimadzu), una estufa FANEM; un vortex - Genie 2 y una balanza analítica Accculab Sartorio Group.

\section{ANÁLISIS:}

\section{Evaluación del efecto antioxidante}

Para la determinación de la actividad antioxidante se aplicó el método DPPH, el cual consistió en evaluar la potencia antioxidante mediante el barrido de radicales libres de la muestra de análisis y del registro de la densidad óptica de los radicales DPPH.

La actividad antioxidante $\mathrm{o}$ anti radical se midió mediante el descenso en la absorbancia a $517 \mathrm{~nm}$ según método de Brand Williams, modificado por Molyneux y Suarez. El blanco se preparó con metanol agua 2:1 para ajustar el espectrofotómetro a cero. Además, el blanco de muestra se preparó con $0,75 \mathrm{ml}$ de muestra (solución A) y $1,5 \mathrm{ml}$ de metanol. El patrón de referencia se preparó con 1,5 ml de DPPH y $0,75 \mathrm{ml}$ de agua destilada. Se pesó $2 \mathrm{mg}$ de DPPH (2,2-difenil-1-picrilhidrazilo) y se disolvió en $100 \mathrm{ml}$ de metanol $(2 \mu \mathrm{g} / \mathrm{ml})$. Se pesó $12 \mathrm{mg}$ de muestra pulverizada seca y se disolvió en $20 \mathrm{ml}$ de metanol (solución A), luego se procedió a realizar disoluciones para obtener concentraciones de $200 \mu \mathrm{g} / \mathrm{ml}, 100 \mu \mathrm{g} / \mathrm{ml}, 50 \mu \mathrm{g} / \mathrm{ml}, 25$ $\mu \mathrm{g} / \mathrm{ml}, 10 \mu \mathrm{g} / \mathrm{ml}$ y $5 \mu \mathrm{g} / \mathrm{ml}$. Se dejaron por espacio de 30 minutos protegidas de la luz, luego de ese tiempo, se leyeron a $517 \mathrm{~nm}$ en el espectrofotómetro UV/Vis.

\section{Método del cribado fotoquímico}

Se usó el método de cribado fitoquímico de Olga Lock (1994) (27), Es un método colorimétrico, donde se catalogó cualitativamente la presencia del metabolito en “+++" (abundante), “++" (moderado), “+” (leve) y “-" (ausencia). Se evaluó la presencia de los siguientes metabolitos: alcaloides (reactivo Dragendorff, Mayer y Hager), fenoles (reactivo cloruro férrico), quinonas (reactivo Borntranger), esteroides y triterpenos (reactivo Liberman-Buchard).

\section{Análisis de los resultados}

Los resultados se presentan como valores absolutos y relativos, para ello se dispuso del programa Microsoft Excel 2013.

\section{RESULTADOS}

\section{Análisis Fitoquímico}

La marcha fitoquímica de los tres extractos de los capítulos de Tagetes filifolia arrojó abundante contenido de fenoles en el extracto en alcohol etílico y en el extracto en agua destilada, con ausencia en el extracto etéreo; además, contenido moderado de quinonas en el extracto en alcohol etílico, con ausencia en el extracto etéreo y en el extracto en agua destilada; en tanto que en ninguno de los tres extractos se hallaron esteroides, triterpeno, ni alcaloides (Tabla 1). 
Tabla 1. Screening fitoquímico de los capítulos de Tagetes filifolia Lag.: Extracto Etéreo, extracto en alcohol etílico y extracto en agua destilada

\begin{tabular}{|l|c|c|c|}
\hline Identificación de metabolitos & & Resultados \\
\hline Reactivos & EE & EAE & EAD \\
\hline Fenoles: Cloruro férrico & - & +++ & +++ \\
\hline Quinonas: Borntranger & - & ++ & - \\
\hline Esteroides y/o triterpenos: Liberman-Buchard & - & - & - \\
\hline Alcaloides: Dragendorff & - & - & - \\
\hline \multicolumn{1}{|}{ Mayer } \\
$\quad$ Wagner & - & - & - \\
\hline Leyenda: (-) Negativo, (+) Leve, (++) Moderada, (+++) Abundante & - & - \\
\hline
\end{tabular}

\section{Capacidad antioxidante}

Para los siguientes gráficos se empleó el ácido ascórbico como sustancia referencial debido a su capacidad ideal de captación de radicales libres. Se encontró que el extracto en alcohol etílico tiene mayor actividad antioxidante a 100 $\mathrm{ug} / \mathrm{ml}(91.26 \%)$ (Figura 1), mientras que el extracto etéreo a $50 \mathrm{ug} / \mathrm{ml}(93.57 \%)$ (Figura 2) y $5 \mathrm{ug} / \mathrm{ml}(27.5 \%)$ (Figura 3).

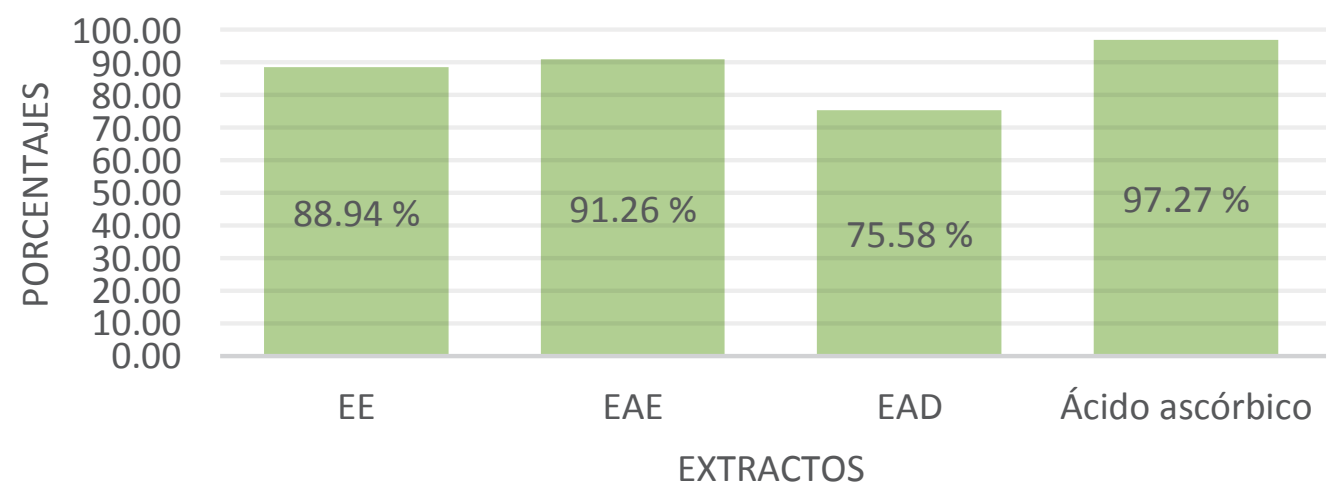

Figura 1. Porcentaje de captación de radicales libres a $100 \mu \mathrm{g} / \mathrm{ml}$. Extracto etéreo, extracto en alcohol etílico y extracto en agua destilada

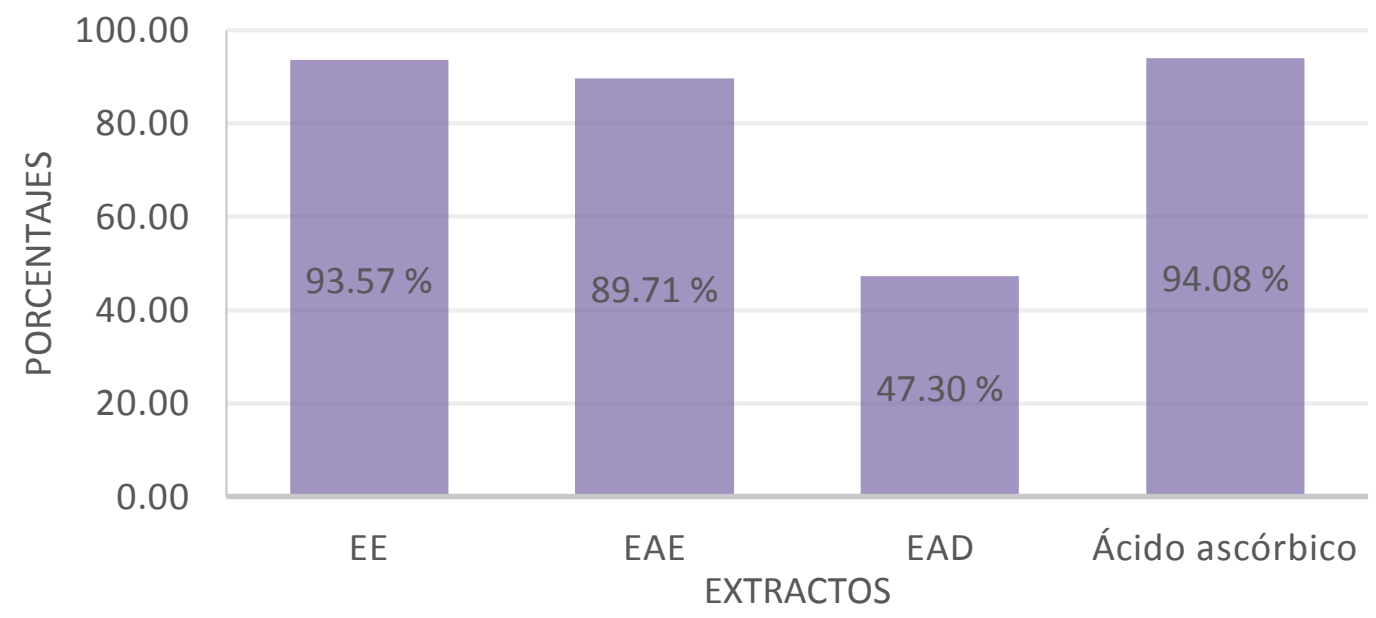

Figura 2. Porcentaje de captación de radicales libres a $50 \mu \mathrm{g} / \mathrm{ml}$. Extracto etéreo, extracto en alcohol etílico y extracto en agua destilada 
Actividad antioxidante y marcha fitoquímica de los capítulos de Tagetes filifolia Lag. "pacha anís"

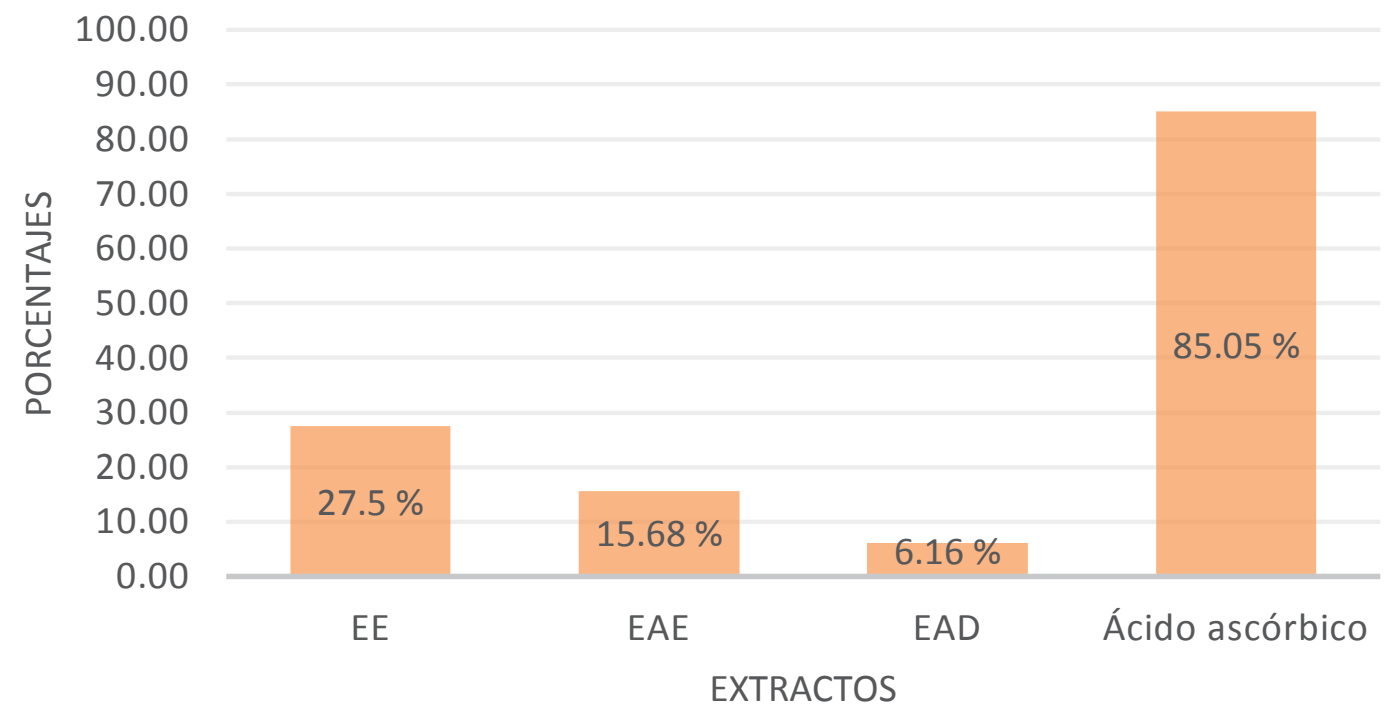

Figura 3. Porcentaje de captación de radicales libres a $5 \mu \mathrm{g} / \mathrm{ml}$. Extracto etéreo, extracto en alcohol etílico y extracto en agua destilada

\section{DISCUSIÓN}

Nuestro estudio encontró fenoles, tanto en el extracto en agua destilada como en el extracto en alcohol etílico; teniendo este último, cantidades moderadas de quinonas. Un estudio en Riobamba-Ecuador no encontró fenoles (usando $\mathrm{FeCl} 3$ ) en su extracto etanólico de Tagetes filifolia lag., pero si coincidió en la presencia de quinonas (usando la prueba de Borntrager) y ausencia de triterpenos y esteroides (usando la prueba de Lieberman Burchard); hallaron entre otros la presencia de chalconas (usando H2SO4), flavonoides (usando SHINODA), lactonas (usando BALJET), flavonas (usando M. BETOLO), además de lípidos y aceites esenciales (usando SUDAN III) ${ }^{(28)}$. Una explicación al contraste de nuestro estudio con el estudio de Riobamba-Ecuador podría deberse a que Riobomba está 2750 msnm y Matahuasi (sitio de recolección de la planta en nuestro estudio) se encuentra a $3223 \mathrm{msnm}$; originando que tanto por la altura y condiciones climáticas adversas se presenten diferentes metabolitos secundarios $(29,30)$. Nuestro estudio obtuvo resultados parecidos a la cromatografía hecha por la facultad de farmacología de la Universidad Nacional Mayor de San Marcos donde se encontró abundantes compuestos fenólicos; regular cantidad de glucósidos y flavonoides; y pocos alcaloides, quinonas y taninos ${ }^{(15)}$.

Otros autores han realizado el screening fitoquímico del aceite esencial de Tagetes filifolia lag. El estragole fue encontrado en el 95\% del aceite esencial de Tagetes en México (31), mientras que anethole fue encontrado en mayor proporción en Argentina ${ }^{(32)}$. El aceite esencial de la especie peruana de Tagetes filifolia lag., fue estudiado en Cusco y se encontró que los principales componentes fueron cys-anethole (68.2\%) y estragole $(13,7 \%)$; además de isomenthone $(4,5 \%)$, menthone $(4 \%), 1,8$ cigomelone $(1,8 \%)$, y pulegone $(1,1 \%)$. La alta concentración de cysanhetole de esta planta podría explicar su uso tradicional como saborizante ${ }^{(17)}$.

Investigadores de la Universidad Peruana Cayetano Heredia encontraron otros compuestos adicionales como, por ejemplo, trans-anetol (88.2\%) y metil chavicol $(10.9 \%)$ en el aceite esencial de Tagetes filifolia ${ }^{(33)}$. Similares resultados fueron encontrados por otros estudios: el primero llevado a cabo en España, en el Instituto Nacional de Investigación y Tecnología Agraria y alimentaria, el cual encontró que los principales componentes de la Tagetes filifolia fueron anetol (70\%) y metilchavicol $(25.2 \%){ }^{(34)}$; otros estudios encontraron un $73 \%$ de trans-anetol y $23,25 \%$ de estragol ${ }^{(33,35)}$.

Estudios hechos en una variedad de Tagetes (Tagetes minuta, $T$ pusilla y $T$ termiflora) mostró que su aceite esencial contenía flavonoides mayoritariamente agliconas como patuletina, quercetina, quercetagetina, isoramnetina ${ }^{(36)}$.

La composición química de esta planta podría explicar muchos de sus usos tradicionales. En Perú se usa la infusión de las partes aéreas como diurético, anti espasmódico, anti inflamatorio y anti hemorrágico ${ }^{(17)}$. Otra variedad de Tagetes, la Tagetes minuta, ha sido relacionado con tener efecto anti fúngico y antibacteriano; esto podría deberse a la alta concentración de derivados de terpenos en su aceite esencial, el cual generaría inestabilidad de las membranas bacterianas y su posterior efecto antibacteriano ${ }^{(37)}$.

Se ha encontrado que los fenoles, uno de los principales componentes hallados en nuestro estudio, actúan 
como antioxidantes, molusquicidas, antihelmínticos, antihepatotóxicos, antiinflamatorios, antidiarreicos, anti ulceroso, antivirales, antialérgicas y vasodilatadores. En Ecuador se estudió su actividad antimicrobiana y se llegó a la conclusión de que el extracto etéreo de Tagetes minuta L. sí reportaba esta actividad ${ }^{(28)}$.

Se sugiere realizar otros estudios in vitro e in vivo que demuestren la capacidad antioxidante de las flores de Tagetes filifolia Lag., seguido de un estudio químico que permita aislar e identificar los metabolitos activos de la planta en los extractos estudiados.

\section{Actividad Antioxidante}

La capacidad antioxidante de Tagetes filifolia ha sido estudiada en investigaciones previas especialmente en su forma de aceite esencial encontrándose una buena actividad antioxidante ${ }^{(35)}$. En el presente estudio, mediante la prueba $\mathrm{DPPH}$, se halló la capacidad antioxidante del extracto de los capítulos de Tagetes filifolia a una concentración de 100, 50 y $5 \mu \mathrm{g} / \mathrm{ml}$; encontrándose en el extracto en alcohol etílico o etanólico a $100 \mathrm{ug} / \mathrm{ml}$ mayor actividad antioxidante (91.26\%). De forma similar, otra investigación en el extracto etanólico de Tagetes pusilla a una concentración de $100 \mu \mathrm{g} / \mathrm{ml}$, usándose el método del ácido tiobarbitúrico y la formación de ácido úrico, llegó a la conclusión de que existe una actividad antioxidante a esta concentración. Esta actividad antioxidante podría, según los autores, deberse a sus compuestos poli fenólicos ${ }^{(38)}$.

Por otro lado un estudio determinó, a través de la prueba de DPPH para medir la actividad antioxidante del aceite esencial de Tagetes filifolia, un mayor efecto en esta planta a una concentración de $20000 \mu \mathrm{g} / \mathrm{ml}^{(33)}$. Esto rebela un mayor efecto antioxidante del extracto de los capítulos del Tagetes en comparación con su aceite esencial.

Sin embargo un estudio usó el método de decoloración del catión-radical ABTS+ y encontró que el aceite de Tagetes filifolia no tenía capacidad antioxidante ${ }^{(39)}$. Esto se ve reforzado por otro estudio el cual, aplicando el método del 1,1-diphenyl-2-picrylhydrazyl para radicales libres y el B-carotene bleaching test sobre el aceite esencial de Tagetes filifolia, no halló actividad antioxidante ${ }^{(40)}$.

Los procesos inflamatorios y el envejecimiento pueden originar radicales libres como las especies reactivas de oxígeno y la peroxidación de lípidos. Estos radicales libres estas asociados con enfermedades crónicas como son la artritis reumatoide, diabetes, cáncer, entre otros ${ }^{(41-43)}$. La importancia médica del género Tagetes recae en su conocida actividad biológica ${ }^{(43)}$, producto de sus componentes químicos y su actividad antioxidante. Por ejemplo, un estudio hecho sobre Tagetes patulla encontró que los flavonoides extraídos de esta planta, en especial la patuletina, tuvieron propiedades anti artrítica y anti TNF alfa ${ }^{(44)}$.

En conclusión, los mayores metabolitos secundarios encontrados en Tagetes filifolia Lag. fueron los polifenoles y las quinonas. Los extractos de los capítulos disueltos en agua, etanol y éter tienen propiedades antioxidantes a diferentes concentraciones: alcohol etílico a $100 \mathrm{ug} / \mathrm{ml}$ (91.26\%) y el extracto etéreo a $50 \mathrm{ug} / \mathrm{ml}(93.57 \%)$ y $5 \mathrm{ug} /$ $\mathrm{ml}(27.5 \%)$.

Para fines de consumo humano, se recomienda la ingesta de Tagetes en su forma disuelta en agua (infusión) de manera permanente y prolongada, de modo que a largo plazo se consiga mitigar los efectos nocivos de los radicales libres sobre el organismo humano.

\section{REFERENCIAS BIBLIOGRÁFICAS}

1. OMS. Estrategia de la OMS sobre medicina tradicional 20022005 Ginebra. 2002 [Available from: http://whqlibdoc.who. int/hq/2002/WHO_EDM_TRM_2002.1_spa.pdf.

2. Brack E. Tratado de Libre Comercio y Biodiversidad del Perú. Cent Amaz Antropol y Apl Práctica Lima, Perú. 2004.

3. Villarreal Quintanilla JÁ. Familia compositae: tribu tageteae. 2003.

4. Vargas Rodríguez A.P., Bottia Santos E.J. Estudio de la composición de los aceites esenciales de seis especies vegetales cultivadas en los municipios de Bolívar y el peñón - Santander, Colombia. [Tesis de grado de bachiller] Bucaramanga, Universidad Industrial de Santander; 2008.

5. Tropicosorg. Missouri Botanical Garden. 2016 [Available from: http: //www.tropicos.org/Name/2711482.

6. Martínez R, Lenin J. Determinación de la Actividad Antimicrobiana del Extracto Etanólico y Subextractos Etéreo y Clorofórmico de (Drymaria ovatta),(Senna macrophylla) y (Tagetes filifolia Lag). [tesis de Licenciatura]. Ecuador, Escuela Superior Politécnica de Chimborazo. 2013.

7. García-Sánchez F, López-Villafranco M, Aguilar-Rodríguez $\mathrm{S}$, Aguilar-Contreras A. Etnobotánica y morfo-anatomía comparada de tres especies de Tagetes que se utilizan en Nicolás Romero, Estado de México. Botanical Sciences. 2012;90(3):221-32.

8. Barajas JS, Serrato MÁ. Poblaciones silvestres de tagetes filifolia Lag. En el centro-sur de México. Revista Fitotecnia Mexicana. 2006.

9. Fuentes-Cervantes I, Villavicencio-Nieto MÁ, Pérez-Escandón BE. Plantas medicinales de Omitlán, Hidalgo, México. 2013.

10. Hammond GB, Fernández ID, Villegas LF, Vaisberg AJ. A survey of traditional medicinal plants from the Callejón de Huaylas, Department of Ancash, Perú. Journal of Ethnopharmacology. 1998;61(1):17-30.

11. Cruz MAS, Trejo BR, Arenas LO, Gómez AD, Sánchez NG, Martínez FL, et al. Anisillo (Tagetes filifolia Lag.): Recurso Genético Mexicano para controlar la mosquita blanca (Bemicia sp. y Trialurodes sp.). Revista del Jardín Botánico Nacional. 2003;24(1/2):65-70.

12. Camarillo de la Rosa G. Actividad biológica de extractos de Tagetes filifolia Lag. en la mosca blanca Trialeurodes vaporariorum West.(Hemiptera: Aleyrodidae). 2009; 35(2): 177-84

13. Perich MJ, Wells C, Bertsch W, Tredway KE. Toxicity of extracts from three Tagetes against adults and larvae of yellowfever mosquito and Anopheles stephensi (Diptera: Culicidae). Journal of Medical Entomology. 1994;31(6):833-7.

14. Cestari IM, Sarti SJ, Waib CM, Branco Jr AC. Evaluation of the potential insecticide activity of Tagetes minuta (Asteraceae) essential oil against the head lice Pediculus humanus capitis 
Actividad antioxidante y marcha fitoquímica de los capítulos de Tagetes filifolia Lag. "pacha anís"

(Phthiraptera: Pediculidae). Neotropical Entomology. 2004;33(6):805-7.

15. Bonilla P, Lozano N, Arroyo J, Beltrán H. Efecto sobre la gravidez, la prolactina y hormonas sexuales en ratas del sub extracto metanólico de hojas de tagetes filifolia "Anisillo. Ciencia e Investigación. 2008;11(1):7-14.

16. Marotti M, Piccaglia R, Biavati B, Marotti I. Characterization and yield evaluation of essential oils from different Tagetes species. Journal of Essential Oil Research. 2004;16(5):440-4.

17. De Feo V, Della Porta G, Urrunaga Soria E, Urrunaga Soria R, Senatore F. Composition of the essential oil of Tagetes filifolia Lag. Flavour and fragrance journal. 1998;13(3):145-7.

18. Ponte EL, Sousa PL, Rocha MV, Soares PM, Coelho-deSouza AN, Leal-Cardoso JH, et al. Comparative study of the anti-edematogenic effects of anethole and estragole. Pharmacological Reports. 2012;64(4):984-90.

19. Silva-Alves K, Ferreira-da-Silva F, Peixoto-Neves D, VianaCardoso K, Moreira-Júnior L, Oquendo $M$, et al. Estragole blocks neuronal excitability by direct inhibition of $\mathrm{Na}+$ channels. Brazilian Journal of Medical and Biological Research. 2013;46(12):1056-63.

20. Valyova M, Stoyanov S, Markovska Y, Ganeva Y. Evaluation of in vitro antioxidant activity and free radical scavenging potential of variety of Tagetes erecta L. flowers growing in Bulgaria. International Journal of Applied Research in Natural Products. 2012;5(2):19-25.

21. Cerrate E. Manera de preparar plantas para un herbario. UNMSM Museo de Historia Natural, Serie de Divulgación. 1964;1.

22. Ramagosa J RR. Enciclopedia de medicina naturista y alternativa. 2001.

23. Cronquist A. An integrated system of classification of flowering plants: Columbia University Press; 1981.

24. MacBride F. FJ 1936-1971. Flora of Peru Field Museum of Natural History Botanical Series.13(3):1-6.

25. Weberbauer A. El mundo vegetal de los Andes Peruanos: Estudio fitogeográfico. 1945.

26. Tovar Serpa O. Tipos de Vegetación, Diversidad Florística y Estado de Conservación de la Cuenca del Mantaro. 1990.

27. Lock de Ugaz 0 . Investigación fitoquímica. Métodos en el estudio de productos naturales. Lima-Perú Fondo Editorial de la Pontificia Universidad Católica del Perú. 1988.

28. Ortiz U, Ana R. Determinación de la actividad antimicrobiana del extracto etanólico y subextractos etéreo y clorofórmico de Duranta tricanta Juss, Callistemon speciosus y Tagetes minuta L. 2013.

29. Pérez-Urria Carril E, Ávalos García A. Metabolismo secundario de plantas. REDUCA. 2009;2(3):119-45.

30. Dirzo R. Metabolitos secundarios en las plantas, $i$ Atributos panglossianos o de valor adaptativo. Ciencia. 1985;36:137-45.

31. Bohrmann H, Youngken HW. Esdragole, the main compound in the volatile oil of Tagetes filifolia (Compositae). Phytochemistry. 1968;7(8):1415-6.

32. Fester GA, Retamar E, Ricciardi J, Fester A, Martinuzzi E, Retamar J, et al. Aceites esenciales de la República Argentina. 1961.

33. Ruiz C, Cachay M, Domínguez M, Velásquez C, Espinoza G, Ventosilla $P$, et al. Chemical composition, antioxidant and mosquito larvicidal activities of essential oils from Tagetes filifolia, Tagetes minuta and Tagetes elliptica from Perú. Planta Medica. 2011;77(12):30.

34. Maestri DM, Zygadlo JA, Lamarque AL, Labuckas DO, Guzmán CA. Effect of some essential oils on oxidative stability of peanut oil. Grasas y aceites. 1996;47(6):397-400.
35. Muñoz-Acevedo A, Kouznetsov VV, Stashenko EE. Composición y capacidad antioxidante in-vitro de aceites esenciales ricos en Timol, Carvacrol, trans-Anetol o Estragol. Revista de la Universidad Industrial de Santander Salud. 2009;41(3):287-94.

36. Segovia Barrientos IK, Suárez De la Cruz LL. Composición química del aceite esencial de Tagetes elliptica Smith" Chincho" y determinación de su actividad antioxidante, antibacteriana y antifúngica. 2010.

37. García DA, Perillo MA, Zygadlo JA, Martijena ID. The essential oil fromTagetes minuta L. modulates the binding of $[3 \mathrm{H}]$ flunitrazepam to crude membranes from chick brain. Lipids. 1995;30(12):1105-10.

38. De las Heras B, Slowing K, Benedı J, Carretero E, Ortega T, Toledo $\mathrm{C}$, et al. Antiinflammatory and antioxidant activity of plants used in traditional medicine in Ecuador. Journal of ethnopharmacology. 1998;61(2):161-6.

39. Vargas A, Bottia E. Estudio de la composición química de los aceites esenciales de seis especies vegetales cultivadas en los municipios de Bolívar y el peñón-Santander, Colombia. Proyecto de grado, Universidad Industrial de Santander Facultad de Ciencias, Escuela de Química. 2008.

40. Perotti ME SC, Merep AP, Catalán C. A comparative study of antioxidant activity of essential oils from four species of tagetes. BIOCELL. 2007, 31(2): 247-317

41. Raghuveer R, Sreeja K, Sindhuri T, Kumar S. Antihyperlipidemic effect of Tagetes erecta in cholesterol fed hyperlipidemic rats. Der Pharmacia Lettre. 2011;3(5):266-70.

42. Arts IC, Hollman PC. Polyphenols and disease risk in epidemiologic studies. The American journal of clinical nutrition. 2005;81(1):317S-25S.

43. Narayana KR, Reddy MS, Chaluvadi M, Krishna D. Bioflavonoids classification, pharmacological, biochemical effects and therapeutic potential. Indian journal of pharmacology. 2001;33(1):2-16.

44. Jabeen A, Mesaik MA, Simjee SU, Bano S, Faizi S. AntiTNF- $a$ and anti-arthritic effect of patuletin: A rare flavonoid from Tagetes patula. International immunopharmacology. 2016;36:232-40.

\section{Fuentes de financiamiento:}

Este artículo ha sido financiado por los autores.

\section{Conflictos de interés:}

Los autores declaran no tener ningún conflicto de interés.

\section{Correspondencia:}

Bertha Loja Herrera

Dirección: Av. El Corregidor N`1531, Urbanización Los Sirius, Las Viñas, La Molina, Lima-Perú. Centro de Investigación de Medicina Tradicional y Farmacología, FMH -USMP.

Teléfono: 511365 2300, anexo 151-114

Correo electrónico: berta_loja@yahoo.com 